\title{
Value of serum C-reactive protein measurement in the management of bone marrow transplant recipients. Part II: late post-transplant period
}

\author{
SA WALKER, PAMELA G RICHES, TR ROGERS, ${ }^{*} S$ WHITE, ${ }^{*}$ JR HOBBS \\ From the Protein Reference Unit and ${ }^{*}$ Department of Medical Microbiology, Westminster Hospital and \\ Medical School, London SW1P 2AR
}

SUMMARY Seventeen bone marrow recipients transplanted for acute leukaemia (8), chronic leukaemia (1), severe aplastic anaemia (3), and various inborn errors of metabolism (5) had 22 episodes of documented infection in the late ( $>3$ months) post-transplant period. Serum Creactive protein concentrations were considerably increased in patients with bacterial infections, but not in those with viral or fungal infections.

Serum C-reactive protein values were normal in 20 patients transplanted for acute leukaemia (12), chronic leukaemia (1), severe aplastic anaemia (2), and various inborn errors of metabolism (5) who had active chronic graft versus host disease but no evidence of infection. These findings indicate that serum C-reactive protein concentrations are useful in the diagnosis and monitoring of bacterial infections even in the presence of chronic graft versus host disease.

Late infections are arbitrarily defined as those occurring later than three months after transplant. Most of these infections are bacterial,' with an unexpectedly high incidence of serious or fatal pneumococcal disease ${ }^{2}$; they occur more often in bone marrow transplant recipients with active chronic graft versus host disease. ${ }^{3}$ Chronic graft versus host disease occurs in about $30 \%$ of long term survivors of bone marrow transplantation ${ }^{4}$ and is related to a wide range of cellular and humoral immunological abnormalities. ${ }^{5-7}$ This immune imbalance predisposes patients to infection, contributing to morbidity and mortality in the late post-transplant period.

HLA non-identical transplants are associated with an increased risk of late infections,' and the use of the selective immunosuppressant cyclosporin A can be expected to increase the number of such transplants performed.

There is an obvious place for any laboratory test which could be used in the diagnosis and monitoring of bacterial infections to help improve long term survival. In the early transplant period the acute phase reactant $\mathrm{C}$-reactive protein (CRP) is of value

Accepted for publication 29 May 1984 because raised serum concentrations are associated predominantly with bacterial infections and not acute graft versus host disease ${ }^{8}$; but the nature of the acute phase response in chronic graft versus host disease has not been investigated.

We have analysed the serum CRP concentrations found in bone marrow transplant recipients in relation to documented infection and chronic graft versus host disease in the late post-transplant period.

\section{Patients and methods}

Serum CRP concentrations were measured as described previously ${ }^{8}$ on samples taken regularly from bone marrow transplant recipients admitted with suspected or proved infections in the late posttransplant period.

There were 22 clearly documented episodes of infection in 17 patients transplanted for acute leukaemia (8), chronic leukaemia (1), severe aplastic anaemia (3), and various inborn errors of metabolism (5).

CRP concentrations were also measured in serum samples taken in the outpatient clinic from 20 patients transplanted for acute leukaemia (12), chronic leukaemia (1), severe aplastic anaemia (2), 


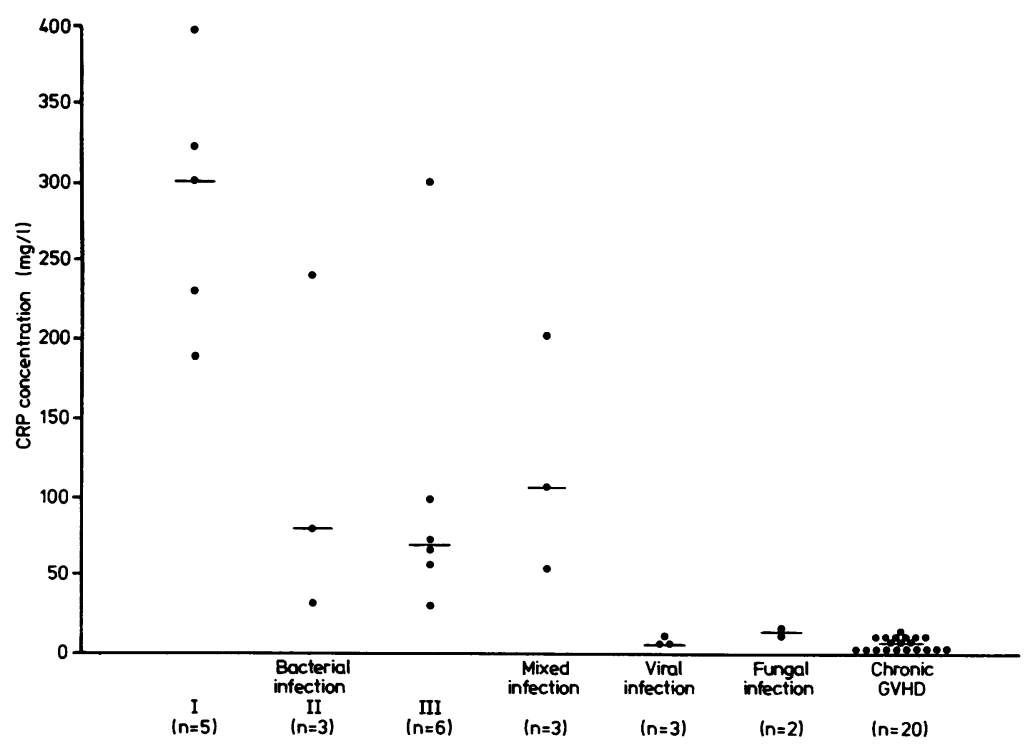

\begin{abstract}
Maximum serum $C$-reactive protein (CRP) concentrations in bone marrow transplant recipients later than three months after transplant.

Bacterial infection: $I$ = microbiologically documented septicaemia; $I I=$ clinically documented infection; III = microbiologically documented localised infection. Horizontal bar indicates median serum CRP concentration for each group.
\end{abstract}

and various inborn errors of metabolism (5) who had active chronic graft versus host disease diagnosed clinically and histologically ${ }^{9}$ but no evidence of infection.

\section{Results}

Patients were grouped according to the type of infection (bacterial, viral, fungal, or mixed). The sites and causative agents (where known) of these infections are shown in Tables 8 and 9 (see appendix), together with the time after transplant and whether or not active chronic graft versus host disease was present.

The maximum serum CRP concentrations reached during each episode of infection are shown in the Figure, together with the serum CRP concentrations in patients with active chronic graft versus host disease without infection and the median CRP value for each patient group. Statistical comparisons were not possible because of the small numbers of patients in some groups. It is quite clear, however, that considerably raised serum CRP concentrations were seen exclusively in association with bacterial infections.

\section{Discussion}

This study has shown that chronic graft versus host disease is not accompanied by increased serum concentrations of the acute phase protein CRP as none of the patients in the group had values above $10 \mathrm{mg} / \mathrm{l}$, the arbitrary upper limit of normal in our laboratory. ${ }^{10} \mathrm{CRP}$ is one of the most sensitive markers of the acute phase response, and in many chronic inflammatory conditions-for example, rheumatoid arthritis, rheumatic fever, Crohn's disease, and familial mediterranean fever-serum concentrations are increased during the active phase of the disease. But in others, such as systemic lupus erythematosus, scleroderma, and mixed connective tissue disease, serum concentrations are normal or only slightly increased in active disease. " The lack of a CRP response in patients with active chronic graft versus host disease is therefore not unique. These patients, like those with systemic lupus erythematosus, are able to mount the expected CRP response to bacterial infections.

Viral and fungus infections were associated with normal serum CRP values, as seen previously in the early transplant period. ${ }^{8}$ 
In conclusion, a raised serum CRP concentration is as sensitive a marker for the diagnosis and monitoring of bacterial infections in bone marrow transplant recipients in the late transplant period as it is in the early transplant period. ${ }^{8}$

We thank members of the Westminster Bone Marrow Transplant team for helpful discussion and access to clinical notes; Dr David Chambers, Stanley Williamson, and Dr R Knight for help in preparing the figures; Professor H Stern, St George's Hospital Medical School, for assistance with virological investigations; and Mrs Jo Payne for preparation of the manuscripts.

\section{References}

' Atkinson K, Farewell V, Storb R, et al. Analysis of late infections after human bone marrow transplantation: role of genotypic non-identity between marrow donor and recipient and of non-specific suppressor cells in patients with chronic graftversus-host disease. Blood 1982;60:714-20.

2 Winston DJ, Schiffman G, Wang DC, et al. Pneumococcal infections after human bone marrow transplantation. Ann Intern Med 1979;91:835-41.

${ }^{3}$ Atkinson K, Storb R, Prentice RL, et al. Analysis of late infections in 89 long-term survivors of bone marrow transplantation. Blood 1979;53:720-31.

${ }^{4}$ Sullivan KM, Shulman HM, Storb R, et al. Chronic graftversus-host disease in 52 patients: adverse natural course and successful treatment with combination immunosuppression. Blood 1981;57:267-76.

s Witherspoon RP, Storb R, Ochs HD, et al. Recovery of antibody production in human allogeneic marrow graft recipients: influence of time post transplantation, the presence or absence of chronic graft-versus-host disease, and antithymocyte globulin treatment. Blood 1981;58:360-8.

${ }^{6}$ Lum LG, Seigneuret MC, Storb RF, Witherspoon RP, Donnall Thomas E. In vitro regulation of immunoglobulin synthesis after marrow transplantation 1. T-Cell and B-Cell deficiencies in patients with and without chronic graft-versus-host disease. Blood 1981;58:431-9.

' Lum LG, Orcutt-Thordarson N, Seigneuret M, Storb R. The regulation of Ig synthesis after marrow transplantation IV. T4 and T8 subset function in patients with chronic graft-versushost disease. J Immunol 1982;129:113-9.

${ }^{8}$ Walker SA, Rogers TR, Riches PG, White S, Hobbs JR. Value of serum $C$ reactive protein measurement in the management of bone marrow transplant recipients. Part I: early transplant period. J Clin Pathol 1984;37:1018-21.

'Sullivan KM, Parkman R. The pathophysiology and treatment of GVHD. Clin Haematol 1983;12:775-89.

${ }^{10}$ Shine B, de Beer FC, Pepys MB. Solid-phase radioimmunoassays for human C-reactive protein. Clin Chim Acta 1981;117:1323.

"Pepys MB. Aspects of the acute-phase response. The C-reactive protein system. In: Lachmann PJ, Peter DK, eds. Clinical aspects of immunology. Oxford: Blackwell Scientific, $1982 ; 50-71$.

Requests for reprints to: Miss SA Walker, Protein Reference Unit, Department of Chemical Pathology, Westminster Hospital, 17 Page Street, London SW1P 2AR, England.

\section{Appendix}

Table 1 Microbiologically documented septicaemia in the early transplant period

\begin{tabular}{ll}
\hline No of episodes & Causative organism(s) \\
\hline 1 & Faecal streptococcus \\
2 & Streptococcus mitis \\
2 & Pseudomonas aeruginosa \\
2 & Klebsiella oxytoca \\
6 & Staphylococcus epidermidis \\
1 & Staph epidermidis \\
& Staph aureus \\
\hline
\end{tabular}

Table 2 Microbiologically documented localised infections (negative blood culture) in early transplant period

\begin{tabular}{ll}
\hline Organism(s) & Associated infection(s) \\
\hline Pseudomonas aeruginosa & $\begin{array}{l}\text { Hickman catheter entry site } \\
\text { Pediastinal abscess }\end{array}$ \\
$\begin{array}{l}\text { Escherichia coli } \\
\text { Streptococcus pneumoniae }\end{array}$ & $\begin{array}{l}\text { Urinary tract } \\
\text { Upper respiratory tract (2) }\end{array}$ \\
$\begin{array}{l}\text { Viridans streptococcus* } \\
\text { Staphylococcus epidermidis }\end{array}$ & $\begin{array}{l}\text { Pneumonia } \\
\text { Cellulitis of foot }\end{array}$ \\
$\begin{array}{l}\text { Staph epidermidis } \\
\text { Staph aureus }\end{array}$ & $\left.\begin{array}{l}\text { Hickman catheter entry site (2) } \\
\text { Str pneumoniae } \\
\text { Haemophilus infuenzae }\end{array}\right\}$ \\
$\left.\begin{array}{l}\text { Faecal streptococcus } \\
\text { Staph epidermidis }\end{array}\right\}$ & $\left.\begin{array}{l}\text { Obscess on arm } \\
\text { Klebsiella oxytoca } \\
\text { Staph epidermidis }\end{array}\right\}$ \\
\hline
\end{tabular}

*Importance uncertain.

Numbers in parentheses $=$ numbers of patients.

Table 3 Clinically documented infection (negative bacteriology) in the early transplant period

\begin{tabular}{ll}
\hline Infection & Outcome \\
\hline $\begin{array}{l}\text { Pyogenic abscess at lumbar } \\
\text { puncture site }\end{array}$ & $\begin{array}{l}\text { Responded to laminectomy and } \\
\text { drainage } \\
\text { Pneumonia }\end{array}$ \\
$\begin{array}{l}\text { Cood response to antibiotics } \\
\text { left zygoma }\end{array}$ \\
$\begin{array}{l}\text { Patient died after initial } \\
\text { response to antibiotics } \\
\text { Clinical evidence only. } \\
\text { Patient died }\end{array}$ \\
\hline
\end{tabular}

Table 4 Viral infections in the early transplant period

\begin{tabular}{|c|c|}
\hline Agent(s) & Associated infection(s) \\
\hline $\begin{array}{l}\text { Rotavirus } \\
\text { Herpes simplex virus } \\
\text { Non A, non B hepatitis virus } \\
\text { Calicivirus } \\
\text { Varicella-zoster }\end{array}$ & $\begin{array}{l}\text { Gastroenteritis (3) } \\
\text { Mucositis (3) } \\
\text { Hepatitis } \\
\text { Gastroenteritis } \\
\text { Chickenpox }\end{array}$ \\
\hline $\left.\begin{array}{l}\text { Cytomegalovirus } \\
\text { Adenovirus } \\
\text { Herpes simplex virus } \\
\text { Parainfluenza } \\
\text { Adenovirus }\end{array}\right\}$ & $\begin{array}{l}\text { Pneumonia; gastroenteritis; } \\
\text { pharyngitis } \\
\text { Pneumonia; conjunctivitis }\end{array}$ \\
\hline
\end{tabular}

Numbers in parentheses $=$ numbers of patients. 
Table 5 Fungal infections in the early transplant period

\begin{tabular}{|c|c|}
\hline $\operatorname{Agent}(s)$ & Associated infection(s) \\
\hline $\left.\begin{array}{l}\text { Candida albicans } \\
\text { Cand albicans } \\
\text { Cand albicans } \\
\text { Aspergillus fumigatus } \\
\text { Asp fumigatus } \\
\text { Cand albicans }\end{array}\right\}$ & $\begin{array}{l}\text { Mucositis (2) } \\
\text { Gastrointestinal } \\
\text { Hickman catheter entry site } \\
\text { Sinusitis } \\
\text { Systemic mycosis }\end{array}$ \\
\hline
\end{tabular}

Number in parentheses $=$ number of patients.

Table 6 Mixed infections in the early transplant period

\begin{tabular}{|c|c|}
\hline Agent(s) & Associated infection(s) \\
\hline $\left.\begin{array}{l}\text { Serratia spp } \\
\text { Cytomegalovirus }\end{array}\right\}$ & $\begin{array}{l}\text { Septicaemia; pneumonia } \\
\text { Septicaemia; pneumonia } \\
\text { Hickman catheter entry site; } \\
\text { mucositis } \\
\text { Vesicles on dorsal trunk } \\
\text { Upper respiratory tract infection; } \\
\text { vesicles on jaw } \\
\text { Urinary tract infection; } \\
\text { mucositis } \\
\text { Urinary tract infection; } \\
\text { gastroenteritis } \\
\text { Mucositis; gastrointestinal }\end{array}$ \\
\hline
\end{tabular}

*Viral infection indicated by histology at necropsy.

†Importance uncertain.
Table 7 Infections occurring concomitantly with acute graft versus host disease in the early transplant period

\begin{tabular}{|c|c|}
\hline Agent(s) & Associated infection(s) \\
\hline $\begin{array}{l}\text { Streptococcus spp } \\
\text { Staphylococcus epidermidis } \\
\text { Staph epidermidis }\end{array}$ & $\begin{array}{l}\text { Septicaemia ( } 5) \\
\text { Septicaemia } \\
\text { Hickman catheter entry site }\end{array}$ \\
\hline $\left.\begin{array}{l}\text { Staph epidermidis } \\
\text { Proteus mirabilis }\end{array}\right\}$ & Septicaemia \\
\hline Staph epidermidis & Septicaemia \\
\hline Faecal streptococcus & Urinary tract infection \\
\hline Staph epidermidis & Septicaemia \\
\hline Enterobacter cloacae & Septicaemia \\
\hline BCG & BCGosis \\
\hline Pseudomonas aeruginosa & Septicaemia \\
\hline $\begin{array}{l}\text { Unknown-responded to } \\
\text { antibiotics }\end{array}$ & $\begin{array}{l}\text { Upper respiratory tract } \\
\text { infection (2) }\end{array}$ \\
\hline $\begin{array}{l}\text { Unknown-responded to } \\
\text { antibiotics }\end{array}$ & Hickman catheter entry site \\
\hline $\begin{array}{l}\text { Unknown-responded to } \\
\text { antibiotics }\end{array}$ & Septicaemia \\
\hline $\left.\begin{array}{l}\text { Faecal streptococcus } \\
\text { Candida albicans }\end{array}\right\}$ & Bacteraemia; mucositis \\
\hline $\left.\begin{array}{l}\text { Staph epidermidis } \\
\text { Cand albicans } \\
\text { Herpes simplex virus }\end{array}\right\}$ & Septicaemia; mucositis (2) \\
\hline $\left.\begin{array}{l}\text { Pseudomonas aeruginosa } \\
\text { Herpes simplex virus }\end{array}\right\}$ & Urinary tract infection, mucositis \\
\hline $\begin{array}{l}\text { Herpes simplex virus } \\
\text { Rotavirus }\end{array}$ & $\begin{array}{l}\text { Pharyngitis (2) } \\
\text { Gastroenteritis (3) }\end{array}$ \\
\hline Varicella-zoster & $\begin{array}{l}\text { Electroencephalogram } \\
\text { consistent with encephalitis }\end{array}$ \\
\hline Cand albicans & Mucositis \\
\hline
\end{tabular}

Numbers in parentheses $=$ numbers of patients.

Table 8 Bacterial infections in the late post-transplant period

\begin{tabular}{|c|c|c|c|}
\hline Organism(s) & Associated infection & $\begin{array}{l}\text { Months afier } \\
\text { transplant }\end{array}$ & $\begin{array}{l}\text { Active chronic graft versus } \\
\text { host disease }\end{array}$ \\
\hline $\begin{array}{l}\text { Bacteroides fragilis } \\
\text { Enterobacter cloacae } \\
\text { Staphylococcus aureus } \\
\text { Streptococcus pneumoniae } \\
\text { Viridans streptococcus } \\
\text { Str pneumoniae } \\
\text { Str pneumoniae } \\
\text { Str pneumoniae } \\
\text { Haemophilus infuenzae } \\
\text { Staph aureus } \\
\text { Staph epidermidis* } \\
\text { Unknown-responded to antibiotics } \\
\text { Unknown-responded to antibiotics } \\
\text { Unknown-responded to antibiotics }\end{array}$ & $\begin{array}{l}\text { Septicaemia } \\
\text { Pneumonia/septicaemia } \\
\text { Septicaemia } \\
\text { Pneumonia/septicaemia } \\
\text { Septicaemia } \\
\text { Pneumonia } \\
\text { Pneumonia } \\
\text { Pneumonia } \\
\text { Pneumonia } \\
\text { Pneumonia } \\
\text { Pneumonia } \\
\text { Pneumonia } \\
\text { Pneumonia } \\
\text { Upper respiratory tract }\end{array}$ & $\begin{array}{r}17 \\
25 \\
37 \\
6 \\
29 \\
5 \\
9 \\
12 \\
12 \\
84 \\
24 \\
10 \\
5\end{array}$ & $\begin{array}{l}\text { Present } \\
\text { Absent } \\
\text { Absent } \\
\text { Present } \\
\text { Present } \\
\text { Present } \\
\text { Present } \\
\text { Absent } \\
\text { Present } \\
\text { Absent } \\
\text { Absent } \\
\text { Absent } \\
\text { Present } \\
\text { Absent }\end{array}$ \\
\hline
\end{tabular}

*Importance uncertain. 
Table 9 Viral, fungal, and mixed infections in the late post-transplant period

\begin{tabular}{|c|c|c|c|}
\hline Agent(s) & Associated infection(s) & $\begin{array}{l}\text { Months after } \\
\text { transplant }\end{array}$ & $\begin{array}{l}\text { Active chronic graft versus } \\
\text { host disease }\end{array}$ \\
\hline $\begin{array}{l}\text { Hepatitis B virus } \\
\text { Varicella-zoster } \\
\text { Varicella-zoster } \\
\text { Candida albicans } \\
\text { Cand albicans }\end{array}$ & $\begin{array}{l}\text { Hepatitis } \\
\text { Chickenpox } \\
\text { Rash on thigh } \\
\text { Brain abscesses } \\
\text { Mucositis }\end{array}$ & $\begin{array}{r}5 \\
3 \\
4 \\
20 \\
12\end{array}$ & $\begin{array}{l}\text { Present } \\
\text { Present } \\
\text { Absent } \\
\text { Absent } \\
\text { Absent }\end{array}$ \\
\hline $\left.\begin{array}{l}\text { Streptococcus pneumoniae } \\
\text { Cand albicans }\end{array}\right\}$ & Pneumonia/septicaemia; mucositis & 4 & Present \\
\hline $\left.\begin{array}{l}\text { Pseudomonas aeruginosa } \\
\text { Cand albicans } \\
\text { Enterovirus } \\
\text { Coliforms* }\end{array}\right\}$ & $\begin{array}{l}\text { Pneumonia/septicaemia; mucositis; } \\
\text { enteritis }\end{array}$ & 6 & Present \\
\hline $\left.\begin{array}{l}\text { Cand albicans } \\
\text { Herpes simplex virus }\end{array}\right\}$ & Pneumonia; mucositis & 26 & Present \\
\hline
\end{tabular}

*Importance uncertain. 